\title{
Recurrent Idiopathic Catatonia: Implications beyond the Diagnostic and Statistical Manual of Mental Disorders 5th Edition
}

\author{
Stanley N. Caroff, Irene Hurford, Henry R. Bleier, Gregg E. Gorton, E. Cabrina Campbell \\ Philadelphia Veterans Affairs Medical Center and Perelman School of Medicine, University of Pennsylvania, Philadelphia, PA, USA
}

\begin{abstract}
We describe a case of recurrent, life-threatening, catatonic stupor, without evidence of any associated medical, toxic or mental disorder. This case provides support for the inclusion of a separate category of "unspecified catatonia" in the Diagnostic and Statistical Manual of Mental Disorders 5th edition (DSM-5) to be used to classify idiopathic cases, which appears to be consistent with Kahlbaum's concept of catatonia as a distinct disease state. But beyond the limited, cross-sectional, syndromal approach adopted in DSM-5, this case more importantly illustrates the prognostic and therapeutic significance of the longitudinal course of illness in differentiating cases of catatonia, which is better defined in the Wernicke-Kleist-Leonhard classification system. The importance of differentiating cases of catatonia is further supported by the efficacy of antipsychotics in treatment of this case, contrary to conventional guidelines.
\end{abstract}

KEY WORDS: Catatonia; Periodic catatonia; Schizophrenia; Cycloid psychosis; Antipsychotic agents; Neuroleptic malignant syndrome.

\section{INTRODUCTION}

Although catatonia is considered a syndrome associated with numerous conditions, an underlying diagnosis may not be discernible in some cases implying the existence of an independent, idiopathic form of catatonia. We describe a pure case of recurrent, life-threatening, catatonic stupor without evidence of any associated medical, toxic or mental disorder. This case is an important example illustrating that catatonia can occur as an independent disorder, providing support for a separate category of "unspecified catatonia" in the Diagnostic and Statistical Manual of Mental Disorders 5th edition (DSM-5). ${ }^{1)}$ However, beyond the limited cross-sectional, syndromal approach of DSM-5, this case also illustrates the importance of considering the longitudinal course of catatonic symptoms that can occur in some cases and determine diagnosis, prognosis and treatment.

\footnotetext{
Received: December 29, 2014 / Revised: February 2, 2015

Accepted: February 25, 2015

Address for correspondence: Stanley N. Caroff, MD

Philadelphia Veterans Affairs Medical Center, 3900 Woodland

Avenue, Philadelphia, PA 19104, USA

Tel: +1-215-823-4066, Fax: +1-215-823-4610

E-mail: stanley.caroff@va.gov
}

CASE

A 24 year old man presented with stupor, mutism and food refusal. He had a negative personal and family history of mental illness until four years prior to admission. At that time, at the age of 20 years old, he was first hospitalized after being found wandering dazed in the streets. Diagnosed with schizophrenia, catatonic type, he received haloperidol initially but was switched to olanzapine and lorazepam. He recovered and returned home, until about twelve months later when he was again admitted to a local hospital for two months with profound stupor, immobility and inanition. He recovered on a combination of haloperidol and lorazepam.

As an outpatient, haloperidol was switched to olanzapine, but both olanzapine and lorazepam were subsequently discontinued due to side effects of weight gain and sedation, resulting in a recurrence of catatonia and re-hospitalization about ten months after his last discharge. During a complicated admission lasting four months, he underwent sequential trials of olanzapine, risperidone, and lorazepam without any improvement in catatonic symptoms. A trial of electroconvulsive therapy (ECT) was initiated, but despite receiving ten bilateral brief-pulse treatments over one month with an average stimulus energy dosage of 386.2 millicoulombs resulting

(c) This is an Open-Access article distributed under the terms of the Creative Commons Attribution Non-Commercial License (http://creativecommons.org/licenses/by-nc/4.0) which permits unrestricted non-commercial use, distribution, and reproduction in any medium, provided the original work is properly cited. 
in ten seizures lasting an average of 37.9 seconds, he showed no change or improvement in his catatonic symptoms and remained stuporous. He finally recovered on a combination of parenteral haloperidol and lorazepam, and was discharged on oral lorazepam and risperidone with monthly paliperidone injections due to suspected medication non-adherence.

After discharge, he showed no signs of residual psychosis or mood disorder for over eight months and was able to return to employment. During remission, his speech was spontaneous, he showed greater emotional expressiveness and he had no psychomotor retardation. However, his affect was mildly blunted and he had limited insight into his illness. Due to his complaint of lethargy and the possibility that some of his residual symptoms represented drug-related extrapyramidal side effects, oral risperidone and lorazepm were discontinued over the next six months, at which time an acute catatonic episode recurred and he was admitted to our hospital.

On this index admission, he was mute and supine with few spontaneous movements. He had mild cog-wheel rigidity without waxy flexibility or catalepsy. His affect was flat and expressionless. Automatic obedience could be elicited by passively moving his limbs into new positions despite verbal instructions to resist. Stereotypies, mannerisms, grimacing, ambitendency, verbigeration, and echo phenomena were absent. He showed negativistic behavior and was rated 21 on the Bush-Francis catatonia rating scale (BFCRS). ${ }^{2)}$

Extensive laboratory investigations, including serum chemistries, hematology, serology, toxicology, cerebrospinal fluid examination, neuroimaging and electroencephalography, revealed no significant abnormality.

Parenteral lorazepam was titrated up to $10 \mathrm{mg}$ daily, but with no response after two weeks, haloperidol $7.5 \mathrm{mg}$ was injected twice daily with diphenhydramine $50 \mathrm{mg}$. Within one week, full remission was achieved with a BFCRS score of zero. After recovery, he was able to speak spontaneously and he again denied any delusions, hallucinations, or changes in mood during or between catatonic episodes. After discharge, he showed mild psychomotor slowing, affective flattening, and limited insight into his illness, similar to previous remissions, but was able to resume employment.

\section{DISCUSSION}

Our patient experienced recurrent catatonic episodes that remitted with mild residual symptoms, but no under- lying disorder could be ascertained. This case highlights the reclassification of catatonia in DSM-5 and the importance of recognizing a form of catatonia with a recurrent longitudinal course as an independent psychomotor disorder.

Although stupor and catalepsy had been recognized as clinical signs since antiquity, Kahlbaum ${ }^{3)}$ has been given credit for recognizing these and other psychomotor features as part of a symptom complex he named catatonia in his seminal monograph of 1874 . While he described numerous disorders associated with catatonia, Kahlbaum contended that catatonia was a disease state distinct from other diagnoses. In contrast, as reviewed by Ungvari et al., ${ }^{4)}$ Kraepelin's and Bleuler's perception of catatonia primarily as a subtype of chronic schizophrenia became the prevailing view in American psychiatry, which explains why our patient was diagnosed with schizophrenia on several prior admissions.

Historically, as catatonic signs in other medical and psychiatric disorders were rediscovered, the specificity of catatonia for schizophrenia was challenged. ${ }^{4)}$ As a result, catatonia in DSM-5 is no longer linked to schizophrenia as a subtype, and can be invoked as a specifier in other disorders. ${ }^{1)}$ DSM-5 also includes an unspecified category of catatonia designed to facilitate treatment in patients until an underlying condition could be identified. ${ }^{5)}$ However, we previously reported that in $4-46 \%$ of patients diagnosed with catatonia in published case series, no known underlying condition could be identified, ${ }^{6}$ providing support for a separate category of idiopathic catatonia as reviewed by Krishna et $\mathrm{al}^{7)}$ The DSM-5 category of "unspecified catatonia" therefore may become the key site for classifying idiopathic cases of catatonia in which a co-existing disorder cannot be confirmed even after diagnostic investigation, which would be faithful to Kahlbaum's original intent. ${ }^{1,3)}$ Accordingly, we believe the catatonic episodes without an identifiable cause in our patient are best diagnosed in DSM-5 as "unspecified catatonia".

However, the fact that Kahlbaum envisioned catatonia as more than a non-specific, cross-sectional syndrome, and proposed that catatonia has a cyclic, alternating course has been forgotten. ${ }^{3)}$ For example, differences in the longitudinal course of catatonia are not addressed in DSM-5, even though a recurrent course of catatonia in some cases had been well-documented. ${ }^{4,8)}$

Concurrently, an alternative system for diagnosing catatonia was developed by Leonhard, expanding on work by Wernicke and Kleist. ${ }^{9)}$ The emphasis of the Wernicke- 
Kleist-Leonhard (WKL) system on psychomotor symptoms and the longitudinal course of illness is ideal for differentiating patients with recurrent catatonic episodes. The diagnoses in the WKL system pertinent to classifying cases like ours include motility psychosis and periodic catatonia. Motility psychosis presents as a primary disorder of quantitative psychomotor function with hyperkinetic and akinetic episodes in the absence of prominent psychopathology of mood or thought. Patients have a good prognosis with full recovery between episodes. Leonhard defined periodic catatonia as more severe than motility psychosis with patients displaying qualitative changes in motor behavior, e.g., stereotypies, in addition to quantitative changes during acute episodes, and accumulating defects after repeated episodes. ${ }^{9)}$ The prognosis of both of these remitting, cyclical disorders is different from and significantly better than catatonia associated with chronic systematic schizophrenia in the WKL system.

In summary, while the diagnosis of each stuporous episode in our case may be limited to "unspecified catatonia" in DSM-5, a more specific diagnosis is provided by Leonhard's concept of periodic catatonia. In contrast to patients with mood disorders, our patient denied any changes in mood, perceptions or thought. Based on the negative symptoms observed in our patient and his response to antipsychotics, one could argue for the DSM-5 diagnosis of schizophrenia with catatonia as a specifier; however, he never displayed any positive signs of psychosis, or persistent symptoms, or the progressive, chronic deterioration associated with schizophrenia. In addition, we believe that the diagnosis of periodic catatonia is more precise in our case than DSM-5 defined schizophrenia because it conveys both the presentation of symptoms exclusively in the psychomotor dimension, and the pattern of recurrence and remissions which is not typical of schizophrenia. These differences between our case and cases of catatonia associated with schizophrenia are supported by Krishna et al. ${ }^{77}$ who demonstrated that patients with idiopathic catatonia could be differentiated from schizophrenia by having a shorter duration and decreased severity of psychotic symptoms but a higher number and severity of catatonic signs. Furthermore, the efficacy of antipsychotics is not specific for or diagnostic of schizophrenia. In fact, Astrup and Fish ${ }^{10)}$ reported that patients with periodic catatonia showed at least moderate improvement compared with cases of chronic systematic schizophrenia who benefited little from antipsychotic therapy, while other investigators have shown that pa- tients with catatonia associated with recurrent affective psychoses may also respond to antipsychotics. ${ }^{11,12)}$

Akinetic motility psychosis could also be considered as the diagnosis in this case because of his significant recovery during remissions and the lack of qualitative psychomotor features, but we concluded that the mild residual symptoms observed between episodes and need for maintenance medications are more consistent with periodic catatonia. ${ }^{9)}$ However, the diagnosis of motility psychosis cannot be excluded entirely because the residual symptoms observed during remissions could be due at least in part to the use of maintenance medications.

Finally, this case raises questions about treatment of catatonia. Lorazepam by itself was not effective during acute episodes. An adequate course of ECT was conducted during one acute episode without any improvement. On several occasions, use of antipsychotics, including haloperidol, in combination with lorazepam resulted in remission without adverse effects, and appeared to be effective in preventing relapse. Recurrence of acute catatonic episodes and hospitalizations repeatedly followed discontinuation of oral antipsychotics and lorazepam. Paliperidone injections alone did not prevent relapse. On the index admission, a trial of lorazepam alone was ineffective, but complete response was achieved after parenteral haloperidol was administered in combination with lorazepam.

In fact, evidence to guide treatment of periodic catatonia is quite limited. Although benzodiazepines and ECT are first-line treatments and usually dramatically effective in acute stuporous catatonia, some evidence suggests that catatonia is heterogeneous in response to these modalities. For example, Ungvari et $a l^{4,13)}$ found no effect of lorazepam on catatonia in a controlled study of patients with chronic schizophrenia, consistent with reviews of other studies showing limited efficacy of lorazepam or ECT alone in treating catatonia associated with chronic psychotic disorders. ${ }^{14,15)}$ Other agents that have been reported as beneficial in periodic catatonia include lithium, anticonvulsants and thyroxine. ${ }^{8,16)}$

Although effective in our case, treatment of catatonia with antipsychotics is controversial. Evidence from case reports is mixed, with antipsychotics sometimes reducing but other times inducing catatonia, or even precipitating neuroleptic malignant syndrome. ${ }^{4,16,17)}$ Whereas antipsychotics are considered toxic and ineffective in motility psychosis, ${ }^{9,16)}$ Astrup and Fish ${ }^{10)}$ reported that $94 \%$ of patients with periodic catatonia showed at least moderate improvement using older typical antipsychotics. Although 
clinical reports have supported potential benefits of atypical antipsychotics in treating some cases of catatonia, ${ }^{18)}$ Peralta et $a l .{ }^{19)}$ found equivalent efficacy of both atypical and typical antipsychotics in treating catatonia associated with psychotic symptoms.

In conclusion, this case of recurrent, idiopathic catatonic stupor supports both Kahlbaum's concept of catatonia as a distinct disease state and the separate category of "unspecified catatonia" in DSM-5 when used to classify cases without an identifiable, underlying cause. But additionally, beyond the limited, cross-sectional, syndromal approach adopted in DSM-5, the recurrent course of catatonia in this case is best classified as Leonhard's periodic catatonia, and serves as a reminder of the importance of both the longitudinal course of catatonia conceptualized as an independent psychomotor psychosis, and the relevance of the WKL system. We also suggest that by closer examination of the course of catatonia, the best options for treatment, including use of antipsychotics, may eventually be clarified.

\section{REFERENCES}

1. American Psychiatric Association. Diagnostic and statistical manual of mental disorders. 5th ed. Arlington, VA:American Psychiatric Press;2013.

2. Bush G, Fink M, Petrides G, Dowling F, Francis A. Catatonia. I. Rating scale and standardized examination. Acta Psychiatr Scand 1996;93:129-136.

3. Kahlbaum KL. Catatonia. Baltimore, MD:The Johns Hopkins University Press; 1973.

4. Ungvari GS, Caroff SN, Gerevich J. The catatonia conundrum: evidence of psychomotor phenomena as a symptom dimension in psychotic disorders. Schizophr Bull 2010;36:231-238.

5. Tandon R, Heckers S, Bustillo J, Barch DM, Gaebel W, Gur RE, et al. Catatonia in DSM-5. Schizophr Res 2013;150: 26-30.

6. Caroff SN, Mann SC, Campbell EC, Sullivan KA. Epidemiology. In: Caroff SN, Mann SC, Francis A, Fricchione GL, editors. Catatonia: From psychopathology to neurobiology. Washington, D.C.:American Psychiatric Press; 2004. p.15-31.

7. Krishna KR, Maniar RC, Harbishettar VS. A comparative study of "Idiopathic catatonia" with catatonia in schizophrenia. Asian J Psychiatr 2011;4:129-133.

8. Gjessing LR. A review of periodic catatonia. Biol Psychiatry 1974;8:23-45.

9. Leonhard K, Beckmann H. Classification of endogenous psychoses and their differential etiology. 2nd revised and enlarged ed. Wien, New York:Springer Verlag;1999.

10. Astrup C, Fish F. The response of the different Leonhard subgroups of schizophrenia of psychotropic drugs. Folia Psychiatr Neurol Jpn 1964;18:133-40.

11. Muneer A. Aripiprazole in the treatment of refractory mood disorders: a case series. Clin Psychopharmacol Neurosci 2014; 12:157-159.

12. Ng F, Dodd S, Berk M. Atypical antipsychotics for bipolar disorder: overblown or blown over? Clin Psychopharmacol Neurosci 2007;5:53-64.

13. Ungvari GS, Chiu HF, Chow LY, Lau BS, Tang WK. Lorazepam for chronic catatonia: a randomized, doubleblind, placebo-controlled cross-over study. Psychopharmacology (Berl) 1999;142:393-398.

14. Rosebush PI, Maurek MF. Pharmacotherapy. In: Caroff SN, Mann SC, Francis A, Fricchione GL, editors. Catatonia: from psychopathology to neurobiology. Washnigton, D.C.:American Psychiatric Press, Inc.;2004. p.141-150.

15. Gazdag G, Mann SC, Ungvari GS, Caroff SN. Clinical evidence for the efficacy of electroconvulsive therapy in the treatment of catatonia and psychoses. In: Swartz CM, editor. Electroconvulsive and neuromodulation therapies. Cambridge, UK:Cambridge University Press;2009. p.124-148.

16. Jabs BE, Pfuhlmann B, Bartsch AJ, Cetkovich-Bakmas MG, Stöber G. Cycloid psychoses-from clinical concepts to biological foundations. J Neural Transm 2002;109:907-919.

17. Caroff SN, Mann SC, Campbell EC, Sullivan KA. Movement disorders associated with atypical antipsychotic drugs. J Clin Psychiatry 2002;63 Suppl 4:12-19.

18. Van Den Eede F, Van Hecke J, Van Dalfsen A, Van den Bossche B, Cosyns P, Sabbe BG. The use of atypical antipsychotics in the treatment of catatonia. Eur Psychiatry 2005;20:422-429.

19. Peralta V, Campos MS, de Jalon EG, Cuesta MJ. DSM-IV catatonia signs and criteria in first-episode, drug-naive, psychotic patients: psychometric validity and response to antipsychotic medication. Schizophr Res 2010;118:168-175. 